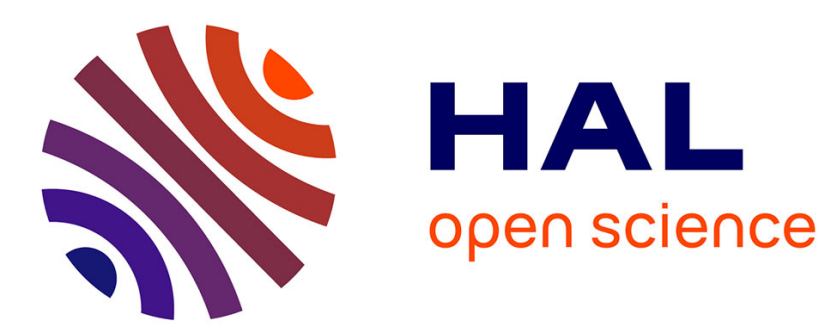

\title{
Les bienséances de l'échange politique. Naissance d'une tribune politique télévisuelle
}

\author{
Eric Darras
}

\section{To cite this version:}

Eric Darras. Les bienséances de l'échange politique. Naissance d'une tribune politique télévisuelle. Politix, 1997, 10 (37), pp. 9-24. 10.3406/polix.1997.1647 . hal-00683068

\section{HAL Id: hal-00683068 https://hal.science/hal-00683068}

Submitted on 27 Mar 2012

HAL is a multi-disciplinary open access archive for the deposit and dissemination of scientific research documents, whether they are published or not. The documents may come from teaching and research institutions in France or abroad, or from public or private research centers.
L'archive ouverte pluridisciplinaire HAL, est destinée au dépôt et à la diffusion de documents scientifiques de niveau recherche, publiés ou non, émanant des établissements d'enseignement et de recherche français ou étrangers, des laboratoires publics ou privés. 


\title{
Éric Darras
}

\section{Les bienséances de l'échange politique. Naissance d'une tribune politique télévisuelle}

In: Politix. Vol. 10, N³7. Premier trimestre 1997. pp. 9-24.

\begin{abstract}
Proprieties of Political Exchange. Birth of a TV Political Forum.

Eric Darras [9-24].

The aims and failures of the first political show (Face a face, 1966-1969) on French television are far from the actual routines of interview news programs (the reverent tone, the design of the settings...). The uncertainties of these beginnings can be seen as the mutual learning - between the main political leaders, journalists and members of the government — of the politically feasible (or speakable) on television. Against the «mediacracy paradigm», this study shows the (nowadays denied) unequal interaction between political guests and journalists «putted in their place», structurally subordinated to the political field. The history of political shows appears like the history of the crystallization of a new political strategic interaction.
\end{abstract}

\section{Résumé}

Les bienséances de l'échange politique. Naissance d'une tribune politique télévisuelle.

Eric Darras [9-24].

Tout oppose les routines télévisuelles actuelles aux ambitions et aux ratés de la première émission politique française : Face-àFace diffusée entre 1966 et 1969. Les balbutiements des premiers commencements peuvent se lire comme l'apprentissage mutuel de la lente délimitation d'un univers des possibles (et du dicible) entre les responsables de l'ORTF, les principaux leaders politiques, les journalistes de presse écrite s'essayant à la télévision et les gouvernants. Contre les explications «médiacratiques», l'étude de cette émission révèle la réalité d'un rapport de force très inégal bien qu'aujourd'hui dénié entre les journalistes, placés en «position d'infériorité structurale» et les professionnels de la politique. L'histoire des magazines politiques de télévision apparaît ainsi comme l'histoire de la cristallisation d'une nouvelle forme d'échange politique.

Citer ce document / Cite this document :

Darras Éric. Les bienséances de l'échange politique. Naissance d'une tribune politique télévisuelle. In: Politix. Vol. $10, N^{\circ} 37$. Premier trimestre 1997. pp. 9-24.

doi : 10.3406/polix.1997.1647

http://www.persee.fr/web/revues/home/prescript/article/polix_0295-2319_1997_num_10_37_1647 


\title{
Les bienséances de l'échange politique Naissance d'une tribune politique télévisuelle
}

\author{
Eric Darras \\ Centre universitaire de recherches \\ administratives et politiques de Picardie \\ Université de Picardie-Jules Verne
}

PPARUE aux États-Unis il y a cent vingt-cinq ans, l'interview
journalistique a d'abord été très critiquée. À la fin du siècle
dernier, elle est encore, pour le rédacteur en chef d'une revue américaine, "ce qu'on a trouvé de mieux jusqu'à présent pour faire du journalisme une activité scandaleuse, offensante pour toutes les narines délicates» ${ }^{1}$. Michael Schudson montre quant à lui combien les journalistes français et anglais répugnent à envisager ce déjà «nouveau journalisme" que consacre la pratique naissante de l'interview, "cette forme moderne et américaine d'inquisition» ${ }^{2}$ assimilée à "l'espionnage», qualifiée de «fumisterie», «d'œuvre de charlatans», d'antichambre de la mort du journalisme... Mais l'emploi du terme «inquisition» ne doit pas tromper. Il s'agit de dénoncer l'impudence ou le coup de force qui consiste à faire interroger les puissants de la terre par ceux qui ne sont "que» journalistes. En aucun cas les observateurs n'incriminent le ton incisif ou le caractère contraignant des questions posées. Bien au contraire, le London Daily News redoute, dès 1869 , avec d'autres journaux européens, que les quotidiens new-yorkais ne mènent la profession de journaliste vers cette sorte de "larbinage" (flunkeying) qu'ils nomment "interviewer" " ${ }^{3}$. De même, G.-K. Chesterton estime plus tard que les interviews américaines restent "généralement très raisonnables" et "toujours très rapides", qu'elles ont ainsi «beaucoup des qualités de l'art dentaire américain» avant d'ajouter que les journalistes s'auto-définissent à l'écrit "comme beaucoup plus bagarreurs (rowdy) et insolents que ne le sont en réalité les interviews»4. Pour l'essentiel, ces commentaires centenaires restent d'actualité.

1. Cité, sans autre référence, par Boorstin (D.), L'image, Paris, UGE, 1971, p. 38-39.

2. New York World, 31 janvier 1871, citê par Schudson (M.), «Question Authority : a History of the News Interview in American Journalism, 1860s-1930s", Media. Culture and Society, 16, 1994, p. 568-569.

3. Ibid., cité p. 569.

4. Ibid., cite p. 580. 
De nos jours, que l'on songe par exemple à L'heure de vérité ${ }^{1}$ ou à 7 sur $7^{2}$, les différents éléments constitutifs du magazine politique apparaissent évidents : chaque mois (ou chaque semaine), un homme politique converse courtoisement autour d'une table avec un ou plusieurs journalistes. Les contraintes de la recherche d'audience ou «les évolutions [liées] du milieu journalistique de l'audiovisuel» 3 semblent dicter les (rares) innovations. Périodiquement sommés de produire du neuf, les professionnels de la télévision bricolent avec de l'ancien et finalement demeurent "dans l'impasse" 4 . En effet, loin de s'expliquer exclusivement par une dépendance croissante aux logiques du spectacle, les produits politiques de la télévision sont, comme d'autres productions symboliques, "déterminés par le passé jusque dans les novations destinées à les dépasser, qui sont inscrites, comme une matrice originelle, dans l'espace des possibles immanent au champ lui-même” ${ }^{5}$. En conséquence, comprendre les échanges «à fleurets à peine mouchetés» ${ }^{6}$ entre Anne Sinclair, Alain Duhamel, François-Henri de Virieu ou Jean-Marie Cavada... et leurs invités politiques respectifs suppose de rappeler l'origine et la formation historique des interviews politiques à la télévision. Pourtant, les quelques sommes historiques consacrées à ce média n'accordent qu'un intérêt tout relatif aux débuts du genre "magazine politique» ${ }^{7}$. Dans ce domaine, le principal frein à l'investigation scientifique reste l'insuffisance de l'archivage.

S'il est vrai que «l'interdépendance des différentes positions de configuration, et l'habitus des hommes ne peuvent s'expliquer et se comprendre que par référence à l'évolution des configurations dont ils sont issus» 8 , c'est bien prioritairement l'étude des premiers commencements qui peut le mieux renseigner sur les causes et les fonctions de cette institution politique et télévisuelle. Ainsi, tout oppose les routines télévisuelles actuelles aux ambitions et aux ratés de la première émission politique française, Face-à-face diffusée entre 1966 et

1. Neveu (E.), «L'Heure de vérité ou le triangle de la représentation», Mots, 20, 1989.

2. Leroux (P.), "Sept sur sept ou la célébration répétée d'une admiration mutuellew, Politix, 23, 1993. Sur ce caractère convenu des échanges politiques à la télévision, voir également : Nel (N.), A fleurets mouchetés. 25 ans de débats télevisés (1960-1980), Paris, INA-La Documentation française, 1988 ; Mouchon (J.), «Le jeu de pouvoir dans les magazines d'information politique», Bulletin du Certeic, 10, 1989.

3. Le Grignou (B.), Neveu (E.), «Intimités publiques. Les dynamiques de la politique à la télévision", Revue française de science politique, $43(6), 1993$, p. 942.

4. Neveu (E.), «Les émissions politiques à la télévision. Les années quatre-vingt ou les impasses du spectacle politique», Hermes, 17-18, 1995.

5. Bourdieu (P.), Raisons pratiques. Sur la theorie de l'action, Paris, Seuil, 1994, p. 77.

6. Dès le second débat politique de l'histoire de la télévision française qui oppose, dans la série des Face a... le 5 décembre 1966, P. Mendes France et R. Schuman, Le Monde remarque : «Politiquement le jeu était subtil. A fleurets à peine mouchetés» (Le Monde, 7 décembre 1966).

7. A décharge, il faudrait rappeler les innombrables difficultés qui s'opposent toujours à l'analyse scientifique de la télevision : obstacles techniques, financiers, juridiques, voire surtout en France, épistémologiques.

8. Elias (N.), Qu'est ce que la sociologie?, Paris, Éditions de l'Aube, 1991, p. 200. 
19691. Et ceci à au moins quatre niveaux : irrégularités des parutions, des dispositifs, des intervenants et du style conversationnel de l'émission. Ce sont là autant de symptômes de l'indétermination caractéristique de cette époque riche en censures gouvernementales multiples. Les balbutiements de la genèse peuvent se lire comme l'apprentissage mutuel de la lente délimitation d'un univers des possibles (et du dicible) entre les responsables de l'ORTF, les principaux leaders politiques, les journalistes de presse écrite s'essayant à la télévision et les gouvernants, tels le ministre de l'information, le Premier ministre et vraisemblablement Charles de Gaulle lui-même qui, selon Georges Pompidou, non seulement regarde la télévision tous les soirs ${ }^{2}$, mais encore veille personnellement au contenu de l'information télévisée ${ }^{3}$. Plus profondément, ces incertitudes sont révélatrices d'un rapport de force très inégal bien qu'aujourd'hui dénié entre les journalistes, placés en «position d'infériorité structurale 4 et les professionnels de la politique : la rencontre télévisuelle d'un journaliste et d'un professionnel de la politique n'est en rien "naturelle" mais s'est au contraire historiquement constituée et au prix fort pour les journalistes. L'histoire des magazines politiques de télévision apparaît ainsi comme l'histoire de la cristallisation progressive d'une nouvelle forme d'échange politique en vue de contrecarrer cette "infériorité congénitale du journaliste" évoquée par Bernard Lacroix 5 .

1. Dès $1954, P$. Corval tente en vain d'institutionnaliser un rendez-vous politique a la télévision avec la série des Faisons le point/Face a l'opinion suivie des Libertés de l'esprit et des Problemes de gouvernement.. Il est possible que l'absence quasi-totale d'archives audiovisuelles ou méme écrites sur ces émissions de la préhistoire télévisuelle ait pour conséquence artefactuelle d'orienter les recherches vers une negation relative de leur importance historique. D'autres émissions font plus tard intervenir occasionnellement des hommes politiques et notamment Faire Face d'E. Lalou et I. Barrère en 1960 et 1962 . Quoi qu'il en soit, contrairement aux experiences politiques précédentes de la télévision, le magazine Face a face bénéficie en 1966 de retombées régulières dans la presse écrite qui consacrent ainsi son élection au rang de producteur d'actualités politiques, ce que remarque J. Bourdon, Haute fidelité, pouvoir et télévision 1935-1994, Paris, Seuil, 1994, p. 88. Voir également : Nel (N.), A fleurets mouchetés, op. cit. ; Nel (N.), Le débat télevise, Paris, Armand Colin, 1990 ; Brochand (C.), Histoire générale de la radio et de la télévision en France, tome 2 (1944-1974), Paris, La Documentation française, 1994.

2. Téle 7 jours, 28 mars 1966.

3. Il n'aurait pas craint, par exemple, d'intimer au ministre de l'Information : "Nessayez pas de persuader les responsables [de la télévision], donnez-leur des instructions. La presse est contre moi. La télévision est à moin ; selon A. Peyreffitte cité par Jeanneney (J.N.), Une histoire des médias des origines a nos jours, Paris, Seuil, 1996, p. 285 . Voir aussi Bourdon (J.), Histoire de la télévision sous de Gaulle, Paris, INA-Anthropos, 1990.

4. Bourdieu (P.), Sur la télévision, Paris, Liber éditions, 1996, p. 53.

5. Lacroix (B.), "A quoi servent les sondages ?", Revue de science administrative de la Méditerranée occidentale, 22-23, 1988, p. 134. Mes remerciements a $D$. Cardon, $P$. Champagne, J. Chevallier, J.-B. Legavre et P. Lehingue ainsi qu'à C. Barbier-Bouvet et ses collègues de l'Inatheque de France. 


\section{Les incertitudes de la genèse}

\section{Une origine gouvernementale}

Si le magazine politique apparaît tardivement à la télévision française, c'est d'abord parce qu'il suppose l'accès de l'opposition au média, c'està-dire la fin du monopole gaullien sur l'expression politique à la télévision. Avec la création d'un magazine à périodicité fixe, les opposants obtiennent une tribune télévisuelle à laquelle ils ne pouvaient préalablement prétendre qu'en période de campagne électorale. De ce point de vue, les quatre premières éditions du magazine sont explicites : Guy Mollet, Valéry Giscard d'Estaing et Waldeck Rochet précèdent la venue du Premier ministre Georges Pompidou. La troisième émission avec le secrétaire général du Parti communiste constitue bien un événement sans précédent dans l'histoire du PCF, comme le consigne L'Humanité : «Ce troisième Face-à-face, c'était d'abord un hommage au communisme, un hommage à notre partis 1 .

Bien que l'expérience de Face-à-face soit rendue possible par une multitude de facteurs convergents dont les pressions politiques de l'opposition (y compris celles de droite et du «centre») et celles de la presse écrite, la conjoncture politique particulière de l'immédiat-aprèsprésidentielle du 19 décembre 1965 où le général de Gaulle est mis en ballottage par François Mitterrand apparaît comme le facteur essentiel. Les scores électoraux de Jean Lecanuet $(15,57 \%$ des suffrages exprimés au premier tour) et François Mitterrand $(32,24 \%)$ ne surprennent guère les spécialistes - Alain Lancelot remarque que François Mitterrand «stabilise l'électorat de gauche au niveau le plus bas qu'il ait atteint depuis trente ans” ${ }^{2}$ - au contraire des journalistes. Le candidat centriste est lui-même loin d'avoir rassemblé sur son nom ne serait-ce que les suffrages exprimés au premier tour des législatives de 1962 en faveur du centre droit (MRP + CNI + Modérés : $19,26 \%{ }^{3}$ ). En réalité, sous le double effet de la percée fulgurante des ventes et locations de téléviseurs et du mythe que constitue l'élection de John-Fitzgerald Kennedy en 1960, cette élection présidentielle pourrait constituer, pour paraphraser Patrick Champagne, le premier succès électoral "de papier» 4 : le triomphe "médiatique» de Jean Lecanuet supposé avoir "crevé l'écran" apparaît largement artefactuel, construit par et pour la presse écrite sommée de se repositionner par rapport à la télévision qui fait ainsi une irruption brutale au sein du cercle politique et médiatique. Certains commentateurs, dont des responsables politiques

1. Cité par Le Monde, 9 mars 1966.

2. Lancelot (A.), «Les résultats de l'élection présidentielle», Projet, 1966.

3. Voir Lancelot (A.), Les élections sous la Ve Republique, Paris, PUF, 1988, p. 34.

4. A propos de «la manifestation de papier»: Champagne (P.), «La manifestation. La production de l'événement politique», Actes de la recherche en sciences sociales, 52-53, 1984. 
de la majorité, concluent à un effet d'usure des ministres trop vus à la télévision au contraire des hommes de l'opposition : ainsi s'expliquerait l'effet de surprise de Jean Lecanuet, «incarnation d'un souffle nouveau". Face à cet effet boomerang (ou underdog), davantage supposé que véritablement démontré, le magazine politique est alors perçu par quelques membres éminents de la majorité comme une solution susceptible de banaliser l'opposition pour mieux la neutraliser. D'emblée, Maurice Denuzière suggère d'ailleurs dans Le Monde cette explication réaliste de la "libéralisation" de la télévision : "Certains membres de l'entourage du général de Gaulle reconnaissent qu'il eût été plus sage de laisser les leaders de l'opposition s'exprimer depuis longtemps devant les caméras. Eux aussi se seraient un peu "usés" et n'auraient peut-être pas obtenu cet effet de surprise qui leur fut finalement favorable»1. Reste qu'on n'en a pas terminé avec cette émission en réduisant l'analyse à cet aspect tactique.

\section{Une espérance de vie aléatoire}

Le premier numéro du premier magazine politique met aux prises le secrétaire général de la SFIO, Guy Mollet et un groupe de journalistes de presse écrite ${ }^{2}$; les émissions suivantes seront conçues sur le même principe, les journalistes étant renouvelés à chaque édition de Face-àface. Ce premier numéro est d'abord retardé avant d'être finalement diffusé le lundi 24 janvier 1966 à 20 heures 30 . À partir du 3 octobre 1966, le magazine mensuel du lundi soir Face-à-face, auparavant produit par Jean Farran, devient En direct avec, placé sous la responsabilité de l'ORTF jusqu'aux «événements» de mai 68, avant de devenir Face à la Presse animé par Michel Droit ; le Premier ministre en est le premier invité le 23 septembre 1968. Mais la survie du magazine reste incertaine et le 18 décembre 1968, en Conseil des ministres, Joël Le Theule, secrétaire d'État à l'Information annonce «la multiplication prochaine des émissions du type Face à la presse». La grille des programmes de l'année 1969 publiée par Télérama prévoit, chaque lundi de 21 heures 15 à 22 heures, la diffusion en alternance des magazines d'informations suivants : Face à la presse, Face-à-face, Face au public et $A u$ fil de l'enquête. Comme leur nom l'indique, chacune de ces émissions correspond à un dispositif particulier, respectivement le débat avec des journalistes, entre hommes politiques, avec des profanes et le reportage. Les Face à... reviennent donc alternativement sous la responsabilité de Henri Marque et Pierre Charpy et celle de l'Actualité télévisée, pour une périodicité initialement prévue de trois émissions par mois. Mais, seules vingt-cinq émissions de la série des Face à... du

1. Le Monde, 14 janvier 1966.

2. Pour cette premiere, les interlocuteurs de l'homme politique sont outre le producteur de l'émission J. Farran (Paris-Match), F. Giroud, co-directrice de l'Express, M. Droit, rédacteur en chef du Figaro littéraire et ex-interlocuteur télévisuel de C. de Gaulle pendant la campagne electorale de 1965 et J. Cau collaborateur régulier de plusieurs journaux (dont France-Observateur, L'Express, Paris-Match, Le Figaro-littéraire) et prix Goncourt (1961). 
lundi, en raison notamment du référendum et de l'élection présidentielle anticipée, seront finalement diffusées du 20 janvier 1969 au 8 décembre 1969 , date de la dernière invitation politique en plateau. Au total, on compte entre une trentaine et une cinquantaine d'éditions du magazine en trois ans, selon que l'on recense ou non les émissions avec reportages et les sujets non politiques. La reconstitution de l'historique des invitations politiques dans la série des Face à... met en évidence cette évolution chaotique de l'émission ${ }^{1}$.

\section{Reconstitution de l'historique des invitations politiques de la série} des Face à...

\section{Face-à-face}

24. 1. 66 : G. Mollet (retardée), ancien président du Conseil, secrétaire général de la SFIO

15. 2. 66 : V. Giscard d'Estaing (et non le $7 / 2$ en raison d'une grève), ministre des Finances démissionnaire

7. 3. 66 : W. Rochet, secrétaire général du Parti communiste

28. 3. 66 : G. Pompidou, Premier ministre

9. 5. 66 : F. Mitterrand, président de la FGDS, ancien ministre, ancien candidat à la présidence de la République

31. 5. $6 \dot{6}$ : E. Faure, ancien président du Conseil, ministre de l'Agriculture

12. 9. 66 : J. Lecanuet (retardée à la demande de l'invité), président du Centre démocrate, ancien candidat à la présidence de la République

\section{En direct avec (même dispositif)}

3. 10. 66 : C. Fouchet, ministre de l'Éducation nationale

14. 11. 66 : M. Debré, ancien Premier ministre, ministre de l'Économie et des Finances - G. Defferre, ancien ministre (G. Defferre étant souffrant ce premier débat politique de l'histoire de la télévision française fut retardé d'une semaine)

5. 12. 66 : M. Schumann, ancien ministre - P. Mendès France, ancien président du Conseil

19. 12. 66 : L. Senghor, président de la République du Sénégal

18. 9. $67: R$. Billères, ancien ministre

13. 11. $67:$ M. Couve de Murville, ministre des Affaires étrangères

18. 12. 67 : G. Mollet, ancien président du Conseil, secrétaire général de la SFIO

29. 1. 68 : J. Chaban-Delmas, ancien ministre

12. 2. 68 : J. Duhamel, président du groupe Progrès et démocratie moderne a l'AN

8. 4. 68 : F. Mitterrand, président de la FGDS, ancien ministre ancien candidat à la présidence de la République

1. Les émissions prévues mais non diffusées ou celles prévoyant un invité non politique ou les émissions de reportages ne sont pas mentionnées. Cet historique est reconstruit par le croisement des fichiers INA (informatique et documentation ecrite) et des informations livrées par Le Monde, Téle 7 jours, Télérama, $\mathrm{N}$. Nel, Le débat télévise (op. cit.) et J. Bourdon, Histoire de la télévision sous de Gaulle (op. cit.). Les incertitudes (sur certaines dates, diffusions, etc.) et plus généralement les difficultés posées à la realisation de l'historique d'une émission vieille seulement d'une trentaine d'années constituent autant d'indices de l'illégitimité persistante de la recherche sur la télévision. 


\section{Face à la presse}

23. 9. 68 : M. Couve de Murville, Premier ministre

2. 12. 68 : R. Pleven, ancien président du Conseil

Face à...

20. 1. 69 : Face-à-face : F.-X. Ortoli (en remplacement de Face au problème cf. infra), ministre de l'Economie et des Finances

(27. 1. 69 : diffusion deux fois retardée de Face à l'événement programmé d'abord le 13/1/69 puis le 20. 1. 69 sous les titres Au fil de l'enquête et Face au probleme : il s'agit d'un documentaire sur "le nouveau parti socialiste" qui intègre plusieurs interviews de leaders socialistes)

3. 2. 69 : Face à la presse : A. Poher, Président du Sénat

10. 2. 69 : Face à l'événement : E. Faure, ancien président du Conseil, ministre de l'Education nationale

17. 2. 69 : Face-à-face : R. Boulin, ministre de l'Agriculture, face aux organisations agricoles J. Deleau (AGPB), M. Debatisse (FNSEA) et J.-B. Doumeng (Inter Agra)

3. 3. 69 : Face à la presse : M. Debré, ancien Premier ministre, ministre des Affaires étrangères

17. 3. 69 : Face-d̀-face : J.-M. Jeanneney, ministre d'Etat

8. 9. 69 : Face à l'opinion : V. Giscard d'Estaing, ancien ministre, président de la FNRI

15. 9. 69 : Face-à-face : trois parlementaires de la majorité s'opposent à trois parlementaires de l'opposition

13. 10. 69 : Face à la presse : J. Fontanet, ministre du Travail, de l'Emploi et de la Population

3. 11. 69 : Face à la presse : O. Guichard, ministre de l'Éducation nationale

10. 11. 69 : Face-à-face : débat sur «la société de tolérance» entre A. Peyrefitte, ancien ministre et $R$. Vadim, cinéaste

1. 12. 69 : Face à la presse : R. Boulin, ministre de la Santé publique et de la Sécurité sociale

8. 12. 69 : Face-à-face : J. Duhamel, ministre de l'Agriculture face aux organisations socio-professionnelles agricoles P. Cormoreche (FNSEA) et M. Simon (CNJA)

\section{Le turn-over des journalistes}

La première de Face-à-face avec Guy Mollet provoque un véritable tollé tant médiatique que politique : à l'instar des premières interviews journalistiques de l'histoire ${ }^{1}$, le comportement des journalistes face au secrétaire général de la SFIO est perçu comme un manquement aux bienséances de l'échange, une infraction aux "règles normatives" régissant les rapports entre les univers politique et journalistique. Citons le commentaire de Télérama : "Nous attendions un débat

1. Au début du siècle, les correspondants americains tentent d'interviewer les chefs d'État européens en arguant des experiences américaines préalables ; les résistances des Europeens ne seront vaincus qu'après la Première guerre mondiale. Ainsi, même si J. Creelman semble avoir été le premier journaliste à obtenir, dès 1897, l'interview d'un Président français (F. Faure - New York Journal, 2 mars 1897, p. 5), W. Abbot se souvient en 1933 que l'idée d'interviewer un chef d'État européen en 1909 semblait encore "ridicule et impossible" (in Schudson (M.), "Question authority...", art. cité, p. 571). 
sérieux : nous avons souvent eu l'impression d'assister à une corrida. Les picadors s'acharnaient sur le taureau solitaire. "Jai eu l'impression de tomber dans un guet-apens, déclara M. G. Mollet... d'être un inculpé... J'ai failli demandé un avocat d'office" [...]. Mais pour que le débat quitte ce ton, il faut que les journalistes sachent eux-mêmes dominer leur passion»1. En 1966, période immédiatement antérieure aux usages journalistiques des sondages, le journaliste ne peut (pas encore) prétendre s'exprimer à la télévision au nom de «l'opinion publique» ${ }^{2}$. Des difficultés sérieuses se posent aussi pour le choix des interlocuteurs journalistiques des invités politiques. Immédiatement rappelé à l'ordre (politique) par la presse écrite, le promoteur de l'émission n'est pas ignorant de l'inégalité du rapport de force improvisé sur le plateau.

De l'aveu même des responsables de la première émission politique française, pour pallier l'inégalité foncière d'une telle interaction, il fallait constituer un groupe de journalistes représentant des tendances politiques opposées à l'invité politique. Jean Farran justifie ainsi le dispositif «seul contre tous» de la première avec G. Mollet : «Deux thèses s'affrontaient : l'une soutenue par quatre personnes, l'autre par une seule. Mais cette arithmétique traduit une inégalité qui n'est pas réelle. Si l'on oppose M. G. Mollet à quatre journalistes qui ne possèdent pas aussi à fond que lui tous les dossiers politiques, ils ne sont pas de force contre lui. Donc, pour qu'il y ait égalité dans cette controverse, il fallait que plusieurs interlocuteurs se trouvent en face de M. G. Mollet» ${ }^{3}$. Ce faisant, le journaliste fait l'étrange aveu d'une infériorité - au niveau de la compétence même : la connaissance des dossiers. Plus encore, il confiera plus tard une inaptitude discursive du journaliste : «Ces personnalités qui viennent sont des champions, si on peut dire du faceà-face. Lorsque Monsieur G. Mollet vient à Face-à-face, il a trente ans derrière lui de réunions électorales, de débats à l'Assemblée, de congrès du PS, d'interpellations. Il a un style, il a une capacité que les journalistes n'ont pas, qui sont souvent des journalistes de plume d'ailleurs"; avant de justifier à nouveau son dispositif : "Alors de ce fait il $y$ a un certain déséquilibre mais nous avons pour nous, nous journalistes un avantage : d'abord nous sommes trois, ensuite entre nous, je dis, entre nous, nous avons préparé les questions alors que celui auquel nous les posons ne les connaît pas du tout et il est donc surpris par nos questions tandis que nous, nous avons eu le temps de les préparer, d'imaginer les réponses qui peuvent venir. Ce qui nous

1. Telérama, 6 février 1966.

2. Champagne (P.), Faire l'opinion. Le nouveau jeu politique, Paris, Minuit, 1990, p. 140. Méme si quelques mois auparavant, l'IFOP vient de réssir le plus gros coup commercial de l'histoire française des instituts de sondages en annonçant sur Europe 1 plusieurs heures avant la télévision le résultat de l'élection présidentielle, l'utilisation du sondage comme arme symbolique de légitimation du journaliste a la télévision n'apparaitra timidement qu'avec l'émission qui succède en 1970 à la série des Face d... : A armes egales.

3. Télé 7 jours, 7 février 1966. 
donne tout de même une position assez forte et qui répartit en partie l'équilibre»1.

La légitimité des journalistes confrontés à un élu ne va donc pas de soi en 1966. Même s'il est intégré au sein d'un groupe de journalistes, une triple condition semble encore nécessaire à celui qui prétend débattre à la télévision avec un homme politique : être reconnu comme une «signature"; être porteur d'une opinion politique clairement attestée ; bénéficier d'un crédit symbolique et politique fort tel que celui né d'un passé résistant.

C'est ainsi qu'en premier lieu, si les journalistes varient d'une édition à l'autre du magazine, ils restent des représentants de la presse écrite ${ }^{2}$. Les journalistes de Face-à-face occupent généralement les plus hautes responsabilités statutaires de ces journaux dont les tirages sont une reconnaissance relative de "représentativité» ${ }^{3}$. Mais l'homme politique est en fait confronté à un groupe de journalistes «dont le trait commun est de ne pas partager toutes les idées de leur interlocuteur selon la formule introductive d'un présentateur occasionnel de l'émission, Jean Lanzi.

En second lieu, le journaliste politique tel qu'il se définit alors est un journaliste politisé, c'est-à-dire engagé, à l'exact opposé du journaliste politique "neutre" et "objectif" tel qu'il se rencontre dès 1970 à la télévision avec $A$ armes égales ${ }^{4}$ : le journaliste est le représentant d'une opinion politique ; en aucun cas celui de «l'opinion publique». La presse écrite des années soixante restant politiquement très différenciée et fréquemment partisane, l'engagement politique des journalistes invités est une qualité nécessaire et revendiquée par le producteur de l'émission. Ainsi s'explique la présence à l'antenne de René Andrieu (rédacteur en chef de L'Humanité depuis 1959, membre du comité central du PCF depuis 1961) puis de quatre autres journalistes de L'Humanité ; de René Dabernat (ancien de Combat, chroniqueur au Monde et à Paris-Match), Jacques Fauvet (Le Monde) face au Premier ministre G. Pompidou ; J. Lecanuet affronte notamment J. Daniel (ancien de L'Express, récent directeur du Nouvel Observateur) et Frédéric Grendel (rédacteur en chef de La Nation - Notre République) ; F.

1. Micros et caméras, 30 avril 1966. Ce commentaire télévisuel «à chaud» du producteur de Face-a-face fait évidemment la très appreciable économie de l'illusion rétrospective. Le succes professionnel du mythe "mediacratique" aidant, un tel discours reflexif a peu de chances d'advenir aujourd'hui, même en sollicitant le «souvenir» des pionniers de la télévision.

2. Le producteur de l'émission (durant neuf mois) lui-même n'est pas un homme de télévision, J. Farran est, au lendemain de la guerre, avocat-stagiaire avant de devenir journaliste au Parisien Liberé (1944-50) puis à Paris-Match.

3. D'ou la présence de représentants de la presse "populaire" : France-Soir, Paris-Jour, L'Aurore.

4. Ce que P. Bourdieu et L. Boltanski avaient d'emblée consignés : «A armes égales : la parade de l'objectivité et l'imposition de problématique», Actes de la recherche en sciences sociales, 2-3, 1976. 
Mitterrand fait face à Pierre Charpy (Paris-Presse-L'intransigeant) et Roger Stéphane, fondateur de France-Observateur en 1950 avant de devenir producteur de télévision.

En troisième lieu, dans la lignée de Pierre Corval (Face à l'opinion), d'Etienne Lalou (Cinq colonnes à la Une, En direct de..., La justice des hommes, Faire face...), de Frédéric Rossif (Cinq colonnes à la Une) ou de Georges Altschuler organisateur sur Europe 1 des premiers débats politiques médiatiques avec Michel Debré et Pierre Mendès France, le passé résistant ou l'engagement aux côtés des Forces françaises sont une caution souvent obligée du journaliste qui interpelle à la télévision l'homme politique. Signe de l'importance de cette légitimité extraite des hauts-faits de la Résistance, F. Mitterrand fait plusieurs fois référence, dans l'édition de Face-à-face du 9 mai 1966, au passé de R. Stéphane dans la Résistance, rappelant notamment qu'ils s'y sont rencontrés. Tout se passe donc comme si l'homme politique d'importance nationale (qui est lui-même généralement à cette époque un ancien résistant ${ }^{1}$ ) à la télévision devait faire face à un journaliste homologue par son action pendant la Seconde guerre mondiale. On compte parmi les journalistes invités de Face à... pour interpeller les hommes politiques au moins douze médaillés de la croix de guerre 39-45 : Henri Amouroux, René Andrieu (également médaille de la Résistance), René Dabernat (également Officier of the Order of the British Empire), J. Daniel, M. Droit (également médaille de la Résistance), J. Fauvet (également Commandeur de la Légion d'honneur), Jean Ferniot, André Frossart, André Guérin (également Commandeur de la Légion d'honneur), Georges Mamy (également rosette de la Résistance), R. Stéphane (également rosette de la Résistance) et Georges Suffert. Il faut encore considérer l'action d'autres journalistes aux cotés de la Résistance : Pierre Drouin, Bernard Lefort, David Rousset, Roger Priouret ou Pierre Sainderichin... Au total, on peut approximativement estimer que les deux-tiers des journalistes invités de la série des Face à... bénéficient, comme quasiment tous les hommes politiques conviés, de cette légitimité d'ancien résistant.

\section{La routinisation d'une relation ambivalente}

Ces modalités de légitimation des journalistes ne suffisent pourtant pas à rendre possible l'institutionnalisation d'un rendez-vous politique à la télévision. Les prétentions des journalistes et des professionnels de la télévision ont été vite rabaissées pour que l'homme politique de «premier rang» concède sa présence sur le plateau, ceci à au moins trois niveaux : le choix du type de participants, le dispositif scénique et les «façons de parler». Ces aménagements opérés, on comprend mieux que

1. Au lendemain de la Liberation, $80 \%$ des députés sont d'anciens résistants actifs selon M. Dogan, "Les filières de la carrière politique en France», Revue française de sociologie, 8, 1967, p. 487. 
les principaux leaders politiques aient pu rapidement «domestiquer» cette tribune naissante du nouveau jeu politique.

\section{La stabilisation des dispositifs}

Selon Télérama, le dispositif originel de Face-à-face reprend «la formule d'une émission célèbre dans le monde entier Meet the Press» ${ }^{1}$. Jérôme Bourdon note que l'information politique américaine constituait bien un modèle de référence pour le SLII (créé dès décembre 1962) comme pour le club Jean Moulin ${ }^{2}$. De même que l'interview journalistique est arrivée en France via les correspondants américains un quart de siècle plus tôt, des hommes politiques français se sont pliés aux règles du jeu télévisuel des magazines politiques américains dès avant que le modèle ne soit importé en France ${ }^{3}$. Meet the Press, le premier magazine politique au monde n'est initialement que la retransmission télévisuelle simultanée à partir de 1947 d'une émission d'interviews radiophoniques de NBC conçue deux ans plus tôt par Lawrence E. Spivak pour promouvoir sa revue l'American Mercury Magazine. Cette formule de l'interview journalistique a été préférée au temps d'antenne dont les partis représentés au Parlement auraient pu bénéficier librement ${ }^{4}$, au débat entre deux professionnels de la politique et à la

1. Télérama, 6 février 1966. Dès 1954, le magazine de P. Corval Face d l'opinion/Faisons le point semble déjà visiblement s'inspirer du dispositif scénique de Meet The Press.

2. Bourdon (J.), Haute fidelite, op. cit., p. 87.

3. On compte ainsi parmi les invités de Meet The Press : P. Mendès France au titre de «Premier of France» (21 novembre 1954); l'ambassadeur de France E. Hervé (3 novembre 1957) également invité de Issues \& Answers et le ministre des Affaires étrangères $M$. Couve de Murville (28 juin 1964). Auparavant, M. Couve de Murville alors ambassadeur de France évoque, le 16 mars 1955, la situation économique en France et la position de la France sur le Vietnam dans Chronoscope, une émission politique diffusée par les stations affiliés de CBS entre 1951 et 1955. Dans cette même émission, H. Bonnet ambassadeur de France discute de l'aide financière américaine et du déclin du communisme en France (20 octobre 1952) tandis que le senateur E. Michelet, ancien ministre des Armées (MRP, 2e cabinet C. de Gaulle en 1945) s'entretient lui du réarmement de l'Allemagne mais aussi des communistes français (25 octobre 1954). D'autres exemples dans Face the Nation notamment pourraient etre cités.

4. Contre le projet défendu par quelques-uns, dont la Fédération syndicale unifié des personnels de l'ORTF, d'une plage horaire télévisuelle laissée en libre accès aux partis ou aux groupes parlementaires (et aux syndicats membres du CES) au prorata de leur representation officielle, c'est le modèle américain du talk show politique qui sera choisi et qui perdurera. Au contraire de la formule finalement retenue, la premiere version laisse les partis de l'opposition libres du choix de leurs porte-parole, mais aussi de leurs mises en scene comme de leurs discours à l'intérieur du temps d'antenne attribue. Or, dans Face-d-face, les journalistes (nombreux) maitrisent le tour et les temps de parole donc l'ordre du jour et d'emblée G. Mollet remarque : "On se trouve finalement face à ses propres opposants et non en opposition politique avec le régime. Je me demande si ce n'était pas concerté. Il ne fallait pas que je puisse parler du général de Gaulle et du gaullisme, non plus que des options socialistes. Pour moi il n'y a eu que dix minutes d'émission, les dix dernières* (Le Monde, 26 janvier 1966). C'est au titre de garants de l'intérét du public et de la qualité des programmes que W. d'Ormesson à l'ORTF et J. Thibau pour la télévision justifieront le choix du magazine politique : "ח n'appartient pas aux responsables de la télévision de décider si des tranches horaires doivent être régulièrement accordées à l'écran à la majorité et à l'opposition. Ce qui est en revanche de notre ressort, c'est le développement d'une information vivante et controversée, et nous devons faire tout ce qui est en notre pouvoir pour éviter que l'information télévisée ne se [suite de la note page suivante] 
confrontation entre l'homme politique et des profanes sur le modèle initié dès 1960 par E. Lalou et I. Barrère avec l'émission Faire Face. Certes, toutes ces formules ont été envisagées par Jacques Thibau, directeur adjoint de la télévision pour la série des Face-à-face ${ }^{1}$. Mais le choix du dispositif étant contraint par les exigences gouvernementales et par celles des invités, celui-ci semblait devoir fédérer l'ensemble des acteurs concernés. La rareté des débats entre hommes politiques obtenus «à l'arraché» dans la série des Face à... témoigne déjà clairement des réticences des professionnels de la politique de «premier rang» face à ce genre télévisuel. Plus subtilement, les leaders politiques de la majorité et de l'opposition ont joué un rôle plus ou moins direct sur le dispositif scénique adopté.

Alors que la mise en scène canonique de l'entretien politique à la télévision (une table, des sièges) apparaît aujourd'hui, aux yeux des analystes eux-mêmes, marqué du sceau de l'évidence, trois dispositifs successifs sont testés pour les trois premières éditions de l'émission politique avant de se fixer sur une formule. En premier lieu, les contradicteurs journalistes et $G$. Mollet sont assis sur des chaises alors que les deux animateurs du débat sont debout ou appuyés sur des colonnes d'un mètre de haut ; tous sont supposés se déplacer dans l'espace, s'exprimer debout mais ils resteront en réalité figés tout au long de l'émission. Dès la deuxième édition, I. Barrère, désireux de forcer le mouvement, supprime les sièges et laisse subsister les colonnes : le ministre démissionnaire du gouvernement V. Giscard d'Estaing est lui-même tantôt debout, tantôt adossé sur la minicolonne. Le premier décor de l'histoire des émissions politiques françaises ne comprend donc pas de table, le second pas de sièges, alors que la version définitive allie table et sièges. Ce dispositif s'est imposé depuis, malgré les réticences de son concepteur et les nombreux défauts qu'il lui trouve déjà : «Face-à-face ne comprend pas de problèmes techniques parce que c'est une émission qui avait pour ambition d'être une série de dialogues libres dans un décor libre avec les mouvements des personnages en toute liberté. Malheureusement à la suite des deux premiers essais, nous nous sommes aperçus que les gens se figeaient dans la position où on les mettait. La première émission avec Monsieur G. Mollet, les personnages étaient assis dans des fauteuils, ils avaient la possibilité de se lever, de s'asseoir sur des cubes. La deuxième émission avec Monsieur Giscard d'Estaing, ils étaient debout au début de l'émission malheureusement on les a retrouvés debout à la fin de l'émission. Pourquoi c'était comme ça ? Parce que nous pensions que cette émission doit avoir une spontanéité qu'aucune autre n'avait... enfin nous le pensions. Les événements ont prouvé que nous avons jusqu'à présent eu tort et que comme vous le voyez, nous en sommes

caractérise d'un cóté par une sorte de journal officiel et de l'autre par des flots de démagogie» (Le Monde, 31 décembre 1965).

1. Le Monde, 31 décembre 1965. 
revenus à la table ronde qui est déjà le prémisse d'un handicap pour cette émission»1. L'échec définitif des premières formules tentées par $I$. Barrère et le choix de la formule la plus «classique» résultent de l'imposition indirecte des volitions des hommes politiques de premier rang qui refusent, en se figeant, la théâtralité voulue par le décor du réalisateur. Les professionnels de la politique subordonneront ensuite leur présence dans les émissions politiques à des conditions optimales de confort donc de sécurité. Le dispositif scénique, aujourd'hui perçu comme «évident» ou "naturel», est en fait celui souhaité initialement par les invités politiques ${ }^{2}$.

Le projet initial prévoit la présence, sur le plateau et en direct, d'une cinquantaine ou d'une centaine de personnes invitées des journalistes ou du leader politique. J. Farran précise d'ailleurs : «Ces spectateurs auront la possibilité de manifester leur présence, ce qui, espère-t-on, créera une ambiance et donnera une garantie supplémentaire d'objectivité" ${ }^{3}$. Le projet est pourtant immédiatement abandonné : il semble que les principaux hommes politiques de l'époque y compris ceux de l'opposition, consultés sur le dispositif, aient préféré le direct, en studio mais sans spectateurs ${ }^{4}$. Certes, l'invité est supposé ne connaître qu'au dernier moment l'identité de ses contradicteurs journalistes et l'émission se retransmet en direct. «Plus rien à voir avec ces tribunes enregistrées d'avance et que l'on modifie avant leur passage à l'antenne si les intéressés ne s'y trouvent pas à leur avantage, si les opinions exprimées paraissent gênantes" s'enthousiasme Jacques Siclier dans Télérama ${ }^{5}$. Toutefois, derrière ces apparentes mises en péril de l'homme politique, les conditions de félicité de sa performance télévisuelle sont en réalité aménagées : J. Farran précise ainsi que les thèmes proposés par l'homme politique sont communiqués aux journalistes huit jours à l'avance ${ }^{6}$ ou que «les détails matériels» sont préalablement convenus avec l'homme politique ${ }^{7}$.

Les intervenants fument à loisir, interposant des volutes de fumée entre les personnage filmés et l'écran. Mais l'essentiel est ailleurs : la mise en scène (et en sens) de l'émission contribue à célébrer l'invité politique. Le générique introduit les intervenants sous un plan panoramique en plongée alors que la musique emprunte au répertoire

1. Micros et caméras . Face-d-face, 30 avril 1966.

2. Dès le 12 juillet 1953 , le président J. Laniel accepte un entretien au journal parlé de la télévision. Dans les années soixante, le dispositif dialogual des conversations au coin du $f e u$, juge plus efficace, remplace les allocutions du Premier ministre. Le modele du aface a la pressen est déjà tellement apprécié par les professionnels de la politique (ou leurs conseillers) qu'en 1965 pendant la campagne électorale le président de la République luiméme choisit de s'entretenir avec $M$. Droit alors que le représentant de l'opposition $F$. Mitterrand dialogue avec G. de Caunes puis R. Louis.

3. Le Monde, 14 janvier 1966.

4. Le Monde, 26 janvier 1966.

5. Telerama, 23 janvier 1966.

6. Télérama, 6 février 1966.

7. Telé 7 jours, 7 février 1966. 
classique dramatisant ; l'extrait tonitruant du Ainsi parlait Zarathoustra de Richard Strauss ('annonce d'un surhomme ?) marque ainsi la césure des programmes vers le sérieux et la gravité du sujet (la politique). La table ronde surélevée par une estrade également circulaire (elle-même «encerclée» par les murs courbes du studio) invite les regards à converger sur l'homme politique invité. Avec une table haute, des chaises rigides et des dossiers posés en évidence, la formule rompt avec les standards de la conversation de salon (le registre, déjà classique, en 1966 de l'émission littéraire) pour se présenter comme une séance de travail. Les différents protagonistes sont disposés dans l'espace selon leurs fonctions respectives : les contradicteurs de l'invité politique sont côte-à-côte et lui font face, alors que le modérateur flanque son invité qui apparaît seul comme doit l'être tout personnage central. De même que la disposition spatiale signifie le rôle et l'importance de chacun, les fonctions et la hiérarchie des protagonistes sont appuyées par des cadrages spécifiques : alors que le réalisateur filme l'invité politique en gros plan voire en très gros plan (la tête occupe tout l'écran), les journalistes sont mis en image par un cadre plus large (plan américain) ou filmés collectivement. L'édition du 9 mai 1966 avec F. Mitterrand présente, pour la première fois, une introduction biographique de l'invité : malgré l'opposition politique avérée de J. Farran à $F$. Mitterrand, le récit de vie politique que le journaliste réalise de son invité emprunte plus à l'hagiographie qu'au simple recensement de ses occupations politiques successives ${ }^{1}$. Ces références enchantées à l'histoire personnelle de l'homme politique deviendront ensuite presque systématiques dans les magazines politiques français et serviront jusqu'à aujourd'hui la célébration du (grand) homme politique ${ }^{2}$.

\section{Les modifications de l'agressivité}

À l'incertitude du décorum correspond l'indétermination du «style» conversationnel. J. Farran précise ainsi : «Il ne s'agit pas à Face-à-face, et ç c'est très important, de faire le procès de quelqu'un [...] ce n'est pas un match pour détruire quelqu'un [...] c'est un dialogue, c'est une controverse un peu chaude pour faire surgir davantage de vérité et d'émotions dramatiques". Cette conjonction de coordination naturalise la coexistence (pourtant problématique) de deux ambitions récurrentes du magazine politique contraint de satisfaire simultanément l'impératif de l'information et celui de la théâtralisation. À la fois «dialogue» et "controverse», l'émission s'oppose pourtant d'emblée au «procès». Le producteur de Face-à-face le précise bien : «I. Barrère et moi-même nous n'avons pas voulu choisir, nous avons voulu que ce soit à la fois une

1. Que ces journalistes ne méconnaissent évidemment pas. Dans cette même émission, $R$. Stéphane pose par exemple la question : «Depuis quand vous considérez-vous comme un homme de gauche, M. Mitterrand ?" (Face-d-face, 9 mai 1966).

2. Darras (E.), «Espaces privés à usages politiques. La psychologisation de la scène politique», in CURAPP, Le for intérieur, Paris, PUF, 1995. 
interview et à la fois une controverse [...] l'interview lui apporte de l'info et la controverse lui apporte de la chaleur». I. Barrère ajoute : «Ce n'est pas un guet-apens [...] ce doit être une controverse et non une bataille [...] il ne faut pas non plus tomber dans une trop grande courtoisie [...] nous sommes toujours sur la lame du couteau. Si vous voulez, nous sommes trop brutaux ou nous sommes trop gentils et pour l'instant je ne peux pas dire que nous ayons trouvé l'axe idéal»1. Progressivement, l'autocensure privilégie des formules et formulations plus classiques, plus euphémisées minimisant d'autant le risque pris par l'homme politique. Avec l'expérience d'une douzaine d'émissions, la politesse et la convivialité s'imposent progressivement. À tel point que de l'aveu même de F. Mitterrand, intimement convaincu que «la télévision est un instrument au service de la propagande gouvernementale", le magazine se caractérise désormais par «une courtoisie et une amabilité peut-être excessives [qui] ont ôté à ces émissions leur véritable caractère» ${ }^{2}$. La civilité devient finalement une caractéristique de l'économie des échanges linguistiques dans les magazines politiques de télévision. Lorsque Guy Mollet revient dans l'émission, Pierre Viansson-Ponté en profite pour faire le point : «ll y a près de deux ans, M. G. Mollet avait eu le redoutable honneur d'inaugurer à la télévision l'émission "Face-àface" devenu depuis "En direct avec...". Ce soir du 24 janvier 1966, les choses s'étaient plutôt mal passées : harcelé, vilipendé par deux au moins de ses interlocuteurs, le leader socialiste avait pris le parti de tendre le dos sous l'orage. Il avait fait quelque peu figure de victime livrée sans défense à ses bourreaux. Depuis bon nombre d'hommes politiques ont affronté à leur tour, avec plus ou moins de bonheur, cette épreuve et l'émission s'est rodée. On ne s'y empoigne plus, on y discute, parfois de façon serrée, et on marque des points dans un climat courtois sinon vraiment détendu [...]. Cette fois tout s'est bien passé» ${ }^{3}$.

\section{Trouver le naturel dans l'artificiel}

Le succès de l'émission aidant ${ }^{4}$, les hommes politiques comprennent immédiatement qu'il leur faut travailler à optimiser leur autorité plastique à la télévision. Des savoir-faire spécifiques s'inventent rapidement. Ainsi, le président E. Faure se fait-il fabriquer des lunettes

1. Micros et cameras . Face-d-face, 30 avril 1966.

2. Télé 7 jours, 8 avril 1968.

3. Le Monde, 20 décembre 1967.

4. Des sondages sont dejà réalisés tous les soirs pour connaitre les réactions du public devant les émissions proposées par la télévision. Pour les deux premiers Face-d-face, les résultats sont les suivants : G. Mollet bénéficie d'une audience de $64 \%$ et d'un indice de satisfaction de 63 ; l'audience de V. Giscard d'Estaing grimpe à $70 \%$ pour un indice de satisfaction de 64 (Le Monde, 19 février 1966). Face-d-face est toutefois programmée a $20 \mathrm{~h} 30$ sur la première chaine. De plus et sans surprise, l'intérét pour l'émission croft regulièrement avec le niveau d'instruction et la position sociale : on compte $39 \%$ de personnes intéressées chez les titulaires d'un CEP pour $65 \%$ au niveau universitaire ; $37 \%$ chez les ouvriers pour $62 \%$ chez les cadres superieurs et les professions liberales (Sondages, 1, 1966, p. 53, cité par Gaxie (D.), Le cens caché. Inegalités culturelles et ségrégation politique, Paris, Seuil, 1978, p. 104). 
spéciales anti-reflets pour comparaître à Face-à-face le 31 mai $1966^{1}$. Télé 7 jours s'interroge encore : "Le raffinement vestimentaire de Jean Lecanuet est bien connu. À la TV, portera-t-il veston croisé ou droit ? Pochette ? De quelle couleur sera sa cravate ? L'homme politique est soucieux de sa bonne tenue» 2 . Les illustrations sont nombreuses. Les hommes politiques ressentent profondément l'épreuve du passage à la télévision vécue comme un événement biographique. L'expérience et l'apprentissage de F. Mitterrand en témoigne : "La difficulté, c'est que malgré les cinq émissions TV de la campagne présidentielle, je n'ai aucune expérience, aucune habitude. Vous avez l'impression d'être dans un laboratoire. Il y a trois caméras, on vous dit "Regardez le voyant rouge". Tout plein de votre propos, vous êtes plongé dans un milieu artificiel, avec caméras, projecteurs, techniciens. Dix personnes indifférentes tournent autour de vous. Les téléspectateurs vous croient seul. Il faut trouver le naturel dans l'artificiel» ${ }^{3}$. De même que vingt ans plus tard, L'heure de vérité est parfois présentée comme un ersatz du grand oral de l'ENA, le professeur de philosophie, Jean Lecanuet «retrouve [dans la préparation de Face-à-face] l'atmosphère de khâgne, ou les élèves sont des disciples questionneurs" ${ }^{4}$ et $\mathbf{E}$. Faure évoque sa leçon d'agrégation. Ces analogies récurrentes comme celles sportives («le match de boxe») ou antiquisantes («l'arène aux lions») célèbrent «l'épreuve» donc l'impétrant, les questionneurs comme les juges (les téléspectateurs dont les critiques de la presse écrite). Autant de bonnes raisons d'éviter leur usage universitaire en qualité de "concepts" scientifiques. Pris comme objet d'étude, ces métaphores condensent et enferment, dès l'origine, la matrice des émissions à venir oscillant entre la contestation affichée et la réalité de la célébration de l'homme politique invité. Les stratégies de présentation de soi télévisuelle font immédiatement l'objet d'anticipations rationnelles et notamment d'entraînements ou de "révisions" préalables (d'où peut-être la référence au concours chez les hommes politiques) qui vont ainsi minimiser le risque, jugé réel, d'une prestation télévisuelle ratée. Ces anticipations stratégiques associées à l'abaissement brutale et durable des prétentions respectives du réalisateur et des journalistes relatives aux dispositifs et au questionnement feront de ceux qui sont présentés (par la presse écrite et l'essayisme) et qui se perçoivent subjectivement comme les «victimes» de la télévision, les «victimes [objectivement] prospères" de L'heure de vérité repérées par Erik Neveu ${ }^{5}$.

1. Téle 7 jours, 31 mai 1966.

2. Téle 7 jours, 2 septembre 1966.

3. Téle 7 jours, 9 mai 1966.

4. Téle 7 jours, 12 septembre 1966.

5. Neveu (E.), “Lheure de verite...", art. cite, p. 68. 